\title{
Development of vocabulary learning application by using machine learning technique
}

\author{
Noor Mohd Ariff Brahin', Haslinah Mohd Nasir², Aiman Zakwan Jidin³, \\ Mohd Faizal Zulkifli ${ }^{4}$, Tole Sutikno ${ }^{5}$ \\ ${ }^{1}$ Centre for Telecommunication Research \& Innovation (CeTRI), Fakulti Kejuruteraan Elektronik dan Kejuruteraan \\ Komputer, Universiti Teknikal Malaysia Melaka, 76100 Durian Tunggal, Melaka, Malaysia \\ ${ }^{1,2,3,4}$ Centre for Telecommunication Research \& Innovation (CeTRI), Fakulti Teknologi Kejuruteraan Elektrik \\ dan Elektronik, Universiti Teknikal Malaysia Melaka, 75450 Ayer keroh, Melaka, Malaysia \\ ${ }^{5}$ Department of Electrical Engineering, Universitas Ahmad Dahlan, Yogyakarta, Indonesia
}

\begin{tabular}{l} 
Article Info \\
\hline Article history: \\
Received Jun 9, 2019 \\
Revised Nov 20, 2019 \\
Accepted Dec 11, 2019 \\
\hline
\end{tabular}

\section{Keywords:}

Android studio

Educational mobile application

Mobile learning

Object detection API

Prediction

TensorFlow

\section{Corresponding Author:}

Noor Mohd Ariff Brahin,

Centre for Telecommunication Research \& Innovation,

Universiti TeknikalMalaysia Melaka,

76100 Durian Tunggal, Melaka, Malaysia .

Email: mohdariff@utem.edu.my

\begin{abstract}
Nowadays an educational mobile application has been widely accepted and opened new windows of opportunity to explore. With its flexibility and practicality, the mobile application can promote learning through playing with an interactive environment especially to the children. This paper describes the development of mobile learning to help children above 4 years old in learning English and Arabic language in a playful and fun way. The application is developed with a combination of Android Studio and the machine learning technique, TensorFlow object detection API in order to predict the output result. Developed application namely "LearnWithIman" has successfully been implemented and the results show the prediction of application is accurate based on the captured image with the list item. The inclusion of the user database for lesson tracking and new lesson will be added for improvement in the future.
\end{abstract}

This is an open access article under the CC BY-SA license.

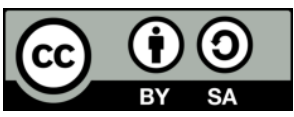

\section{INTRODUCTION}

Mobile devices such as smart phones and tablets are becoming part of human's daily life communication, as well as entertainment. With the advanced of mobile technologies, it can provide an interactive learning content application for children educational purpose which can be simply accessed anywhere and anytime. The fact that children are best learns through playing, the making of an interactive application for Android mobile attracted the research attention. There is a high potential in the usage of mobile technology, in term of how a language can be learned and enhanced. This might be a big help for children to become proficient in second language at early age. Stockwell in his paper shows that the effects of vocabulary learning through mobile platform s are comparable with the traditional ways [1]. This is also supported by Basal et al. through the experiment done that proved the effectiveness of learning vocabulary through mobile applications compared through the traditional paper-based activities [2]. Vocabulary learning is very crucial to build the blocks of language such as read comprehension and language speaking [3]. 
Numerous studies have reported on the use of mobile devices to develop language skills through language learning application in past years. Makoe and Shandu implemented a mobile-based application to develop vocabulary learning mobile app to enhance the English vocabulary teaching and learning [4]. Wang also develop a mobile app to improve the college student's English vocabulary learning with both English and Chinese description [5]. While Sweeney and Moore are investigate the potential of mobile devices to be used in language learning. They found that language learning through mobiles has high potentialbut with the collaboration of both developer and educator to make sure that it can benef it a nd help the students [6]. The most common method for mobile application development using image processing machine learning technique is Mobile Cloud Computing (MCC). MCC is combination of mobiles and cloud computing which can simply processing from the user local devices to the data centre facilities over the internet [7-24]. The major service providers of MCC are including Google, Amazon, Microsoft and Yahoo. Figure 1 shows the general architecture of MCC.

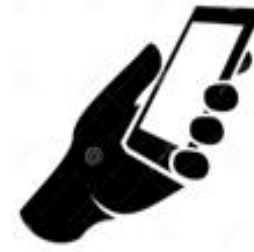

Mobile User

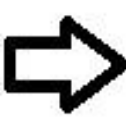

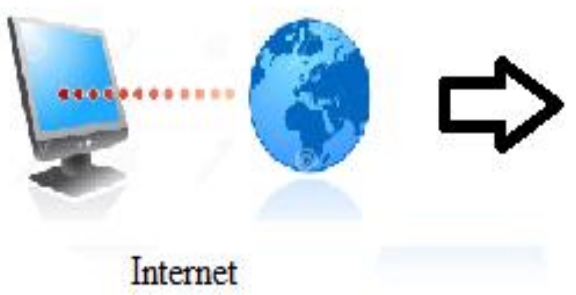

Data centre cloud storage

Figure 1. Mobile cloud computing (MCC) architecture

The users can simply accessing the cloud storage as long as the internet connection is there. However, this can be disadvantage of the MCC. The overall performance of the machine learning can be affected depended on the internet connection speed which it cannot perform inference locally on the device without internet [25-26]. Thus, TensorFlow technique can be a solution to the running machine learning on mobile devices. TensorFlow is able to runs the machine learning inference computation locally on the device. With low latency and small binary size, TensorFlow also can be a lot faster and more reliable than MCC [27-28]. This paper presents the development of Android mobile application namely "LearnWithIman" for language learning through capturing image based on the word given, that aim for toddlers above 4 years old to enhance their English and Arabic vocabulary. TensorFLow deep learning techniques will be used to train the models of captured image for image prediction.

\section{METHOD}

\subsection{Android application development}

In order to attract the children interest, "LearnWithIman" is developed to be a fun application with the learning through playing concept. "LearnWithIman" introduces two basic languages of English and Arabic which divided by different categories. Prior to the application development, the selection of each category is crucial to match the level of the children age. The categories are including the 'Read Easy', 'School Bag', 'Sweet Home', 'Fruits and Veggies', 'Speak Arabic' and 'Exp lore Science'.

For the development of the Android mobile application namely "LearnWithIman", the TensorFlow deep learning technique and Android Studio were used. As mention in Introduction Section, TensorFlow will be used as a machine learning method to predict the image captured from "LearnWithIman" application. "LearnWithIman" application is preloaded with TensorFlow object detection API which uses single shot detector (SSD) with MobileNet pre-trained model that is trained on Microsoft Common Object in Context (COCO) dataset. The captured image will be compared with the preloaded image dataset with the highest score in order to predict the output result. Android Studio is used to customize the look of the application in Android platform. Figure 2 provides the architecture of "LearnWithIman" android mobile application.

Upon entering "LearnWithIman" application, the user is presented with its main interface for categories selection as shown in Figure 3. The application will then display the name of the item with sound that requires the user to capture the image of the item. TensorFlow will perform the prediction by giving the score of the captured image. Once the click button is pressed, the application will compare 
the score with the name of the item. The correct sign will be displayed if the captured image is matched with the name of the item. This will be repeated until all the items are displayed. The point will be shown at the end of application.

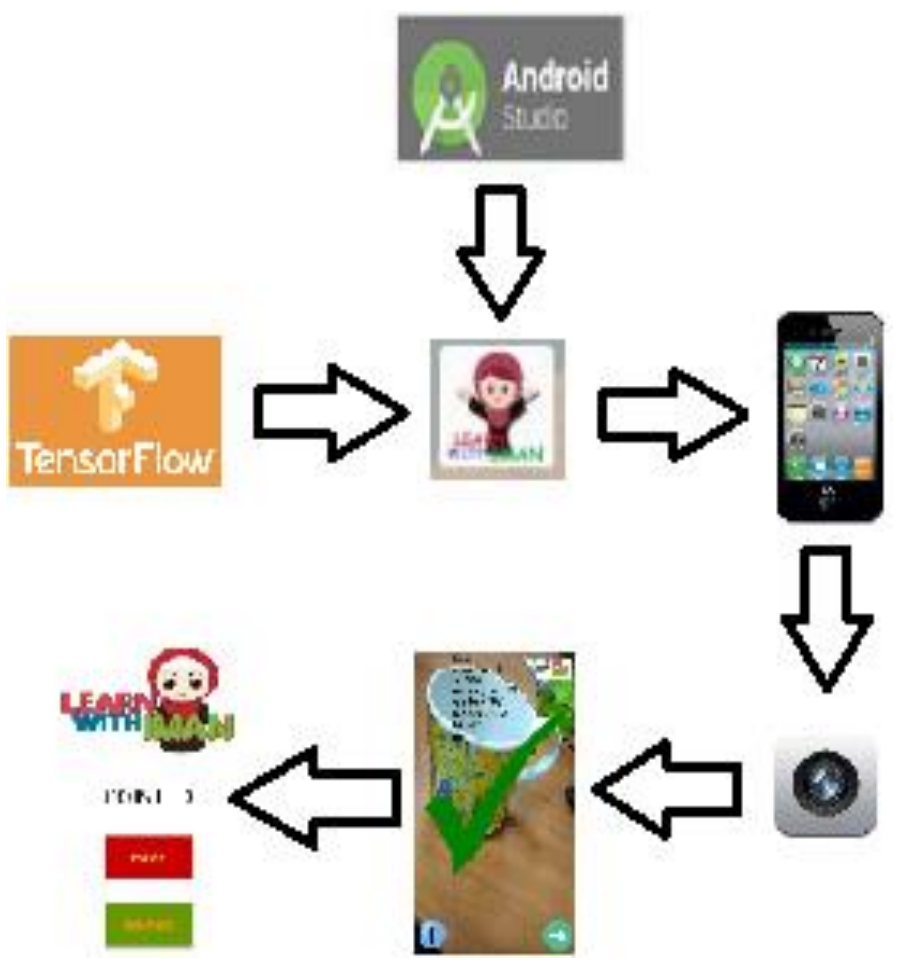

Figure 2. "LearnWithIman" application architecture

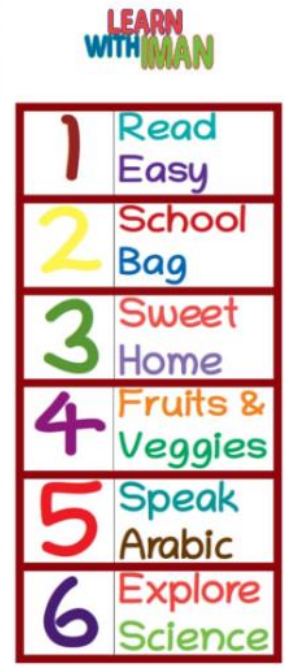

Figure 3. The main interface of "LearnWithIman" application

The Flowchart of 'learnWithIman' application is shown in Figure 4. While Figure 5 presents the screen of image capturing from the application. There are three buttons are available as illustrated in Figure 5, left button is the count of correct item based on the captured image, middle button is to capture the image from the rear camera and the right button is to skip the current item and go to the next item. The name of the item is displayed at the bottom of the application. 


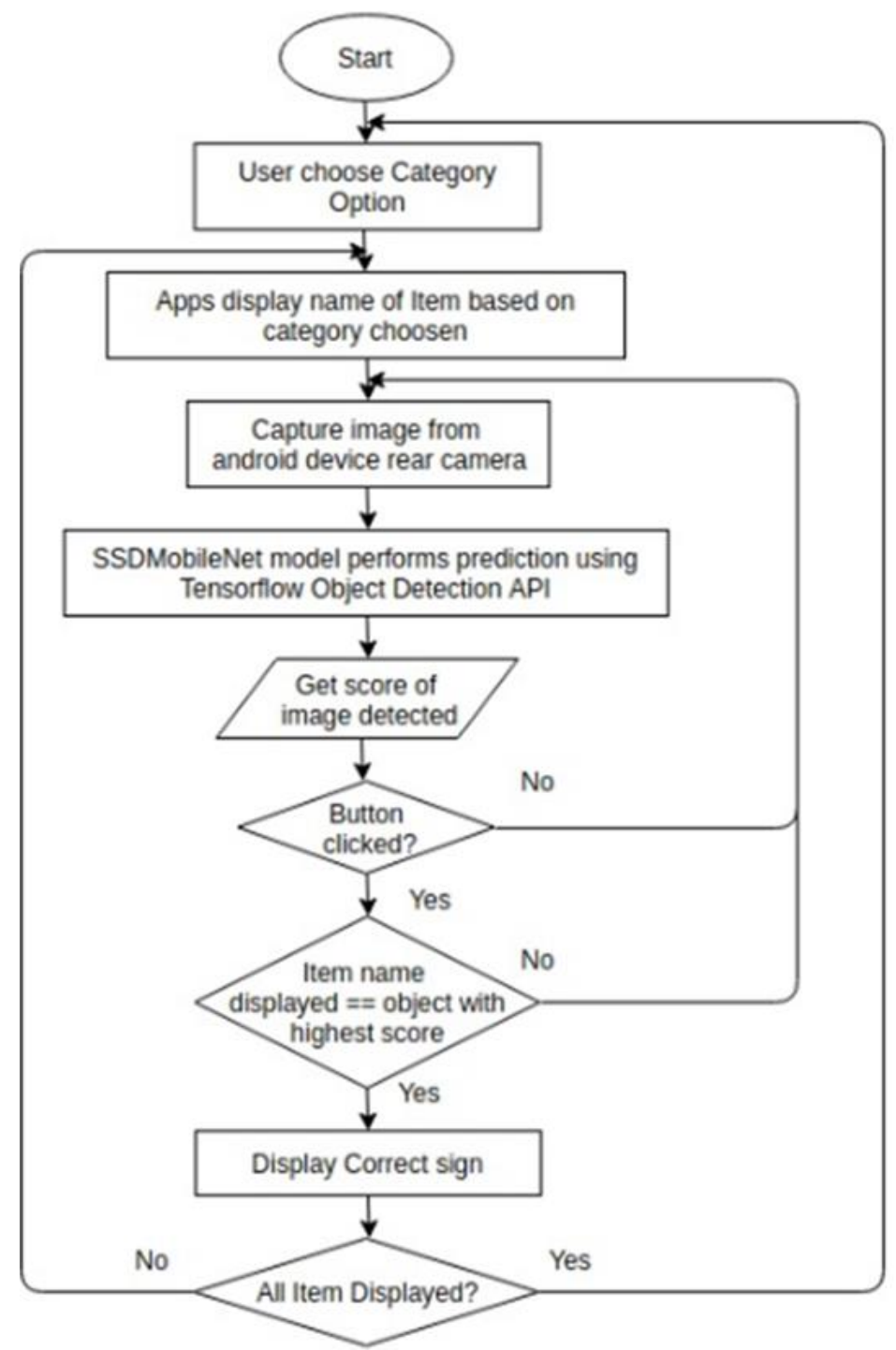

Figure 4. The flowchart of "LearnWithIman" android application

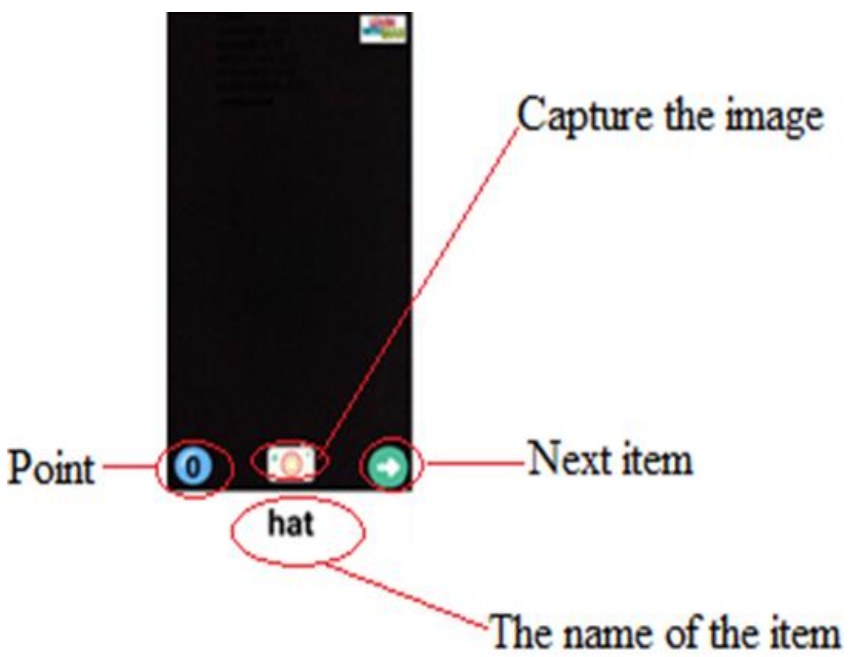

Figure 5. Screen of a pplication to capture the image 


\section{RESULTS AND DISCUSSION}

Several experiments have been done with different requirements to observe the practicability of the application in image detection and prediction. At first, the application was tested with random categories as shown in Table 1. Then, the test for the same item with the different captured image was observed to check the reliability of the application. Last but not least, to see the effect of the image background for the application prediction. Table 1 presents the comparing scores between the captured image and the list item through TensorFlow Object Detection API. As can be seen from the table, "LearnWithIman" is able to detect the captured image accurately according to the list item. On average, the detection time of the captured image took only $100 \mathrm{~ms}$ which considered as sufficient enough.

Table 1. The score of the matched item from different categories

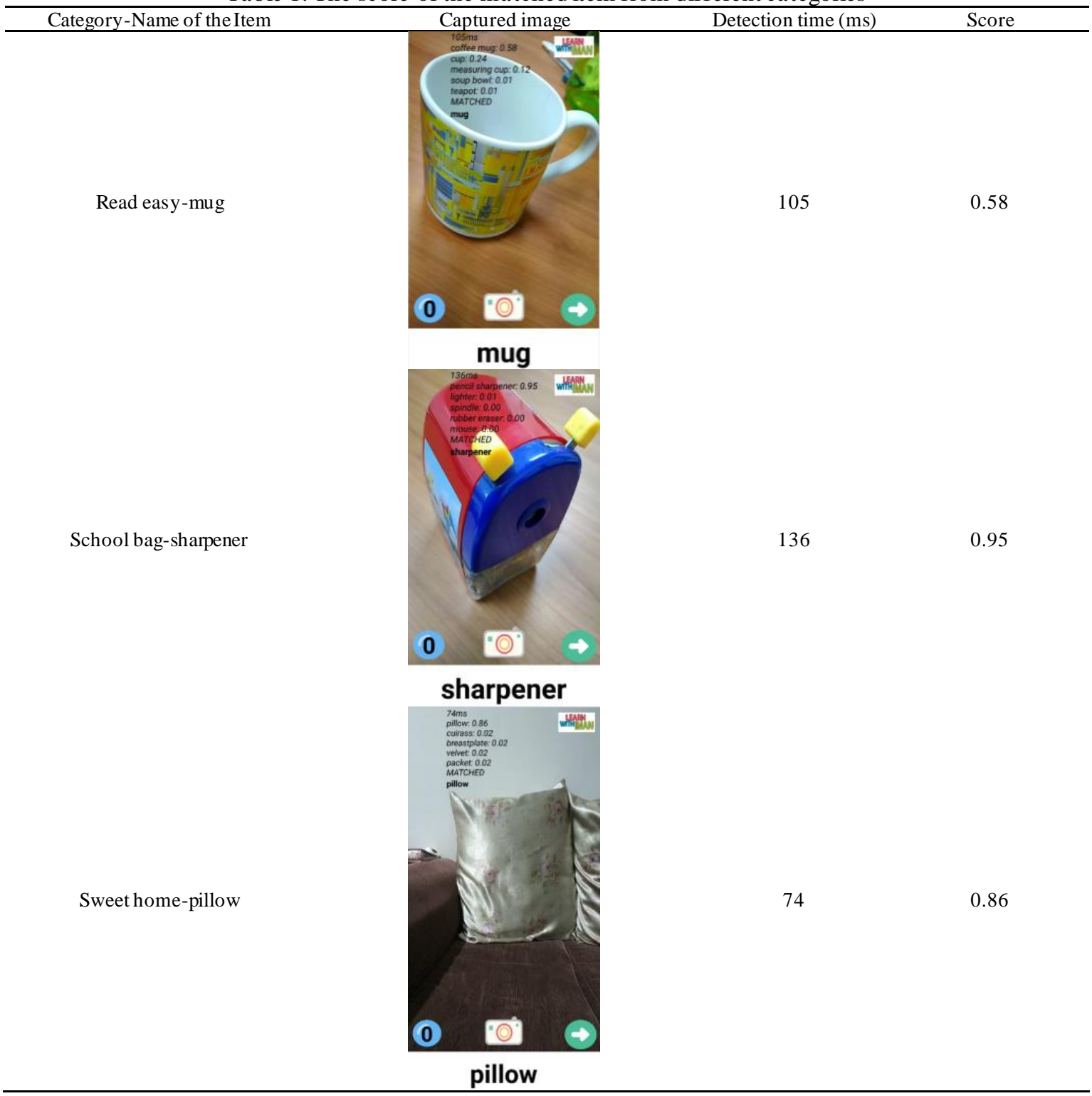

Table 2 illustrates the prediction of the same item but different captured image. It is apparent from the table that "LearnWithIman" consistently matches the item correctly even though the image captured is different. The results shown indicate that the prediction of the application is reliable. Table 3 provides the results of the same item and captured image but with different background. The results obtained revea led that "LearnWithIman" able to detect the matched image even with a different background. 
Table 2. The result from same item with different image

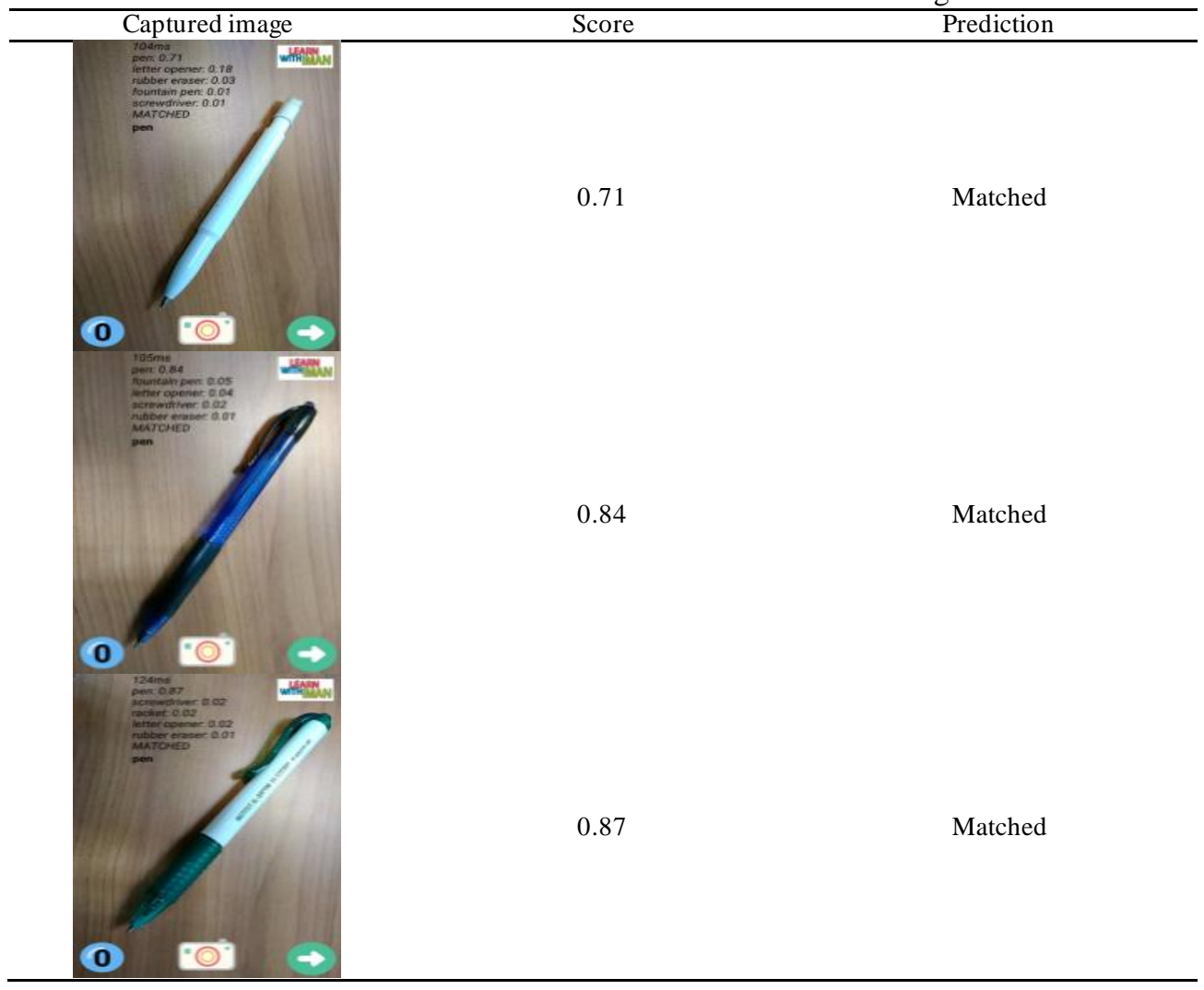

Table 3. The result of same image with different background

Score




\section{CONCLUSION}

This work has presented the development of Android application for children in language learning namely "LearnWithIman". The developed system is able to perform the prediction of the image captured by "LearnWithIman" application for children language learning with high accuracy. The application is implemented with a combination of interactive elements, visual and sound for pronunciation to attract the children interest to learn through mobile application. As for future work, the inclusion of the user database for lesson tracking will be considered. Moreover, new lessons for language learning will be added as well.

\section{ACKNOWLEDGEMENTS}

The authors are grateful for the support received from the Centre for Telecommunication Research \& Innovation (CeTRI), Universiti Teknikal Malaysia Melaka in the accomplishment of this work.

\section{REFERENCES}

[1] G. Stockwell, "Using mobile phones for vocabulary activities: examining the effect of platform," Lang. Learn. Technol., vol. 14, no. 2, pp. 95-110, 2010.

[2] A. Basal, S. Yilmaz, A. Tanriverdi, and L. Sari, "Effectiveness of mobile applications in vocabulary teaching," Contemp. Educ. Technol., vol. 7, no. 1, pp. 47-59, 2016.

[3] Q. Deng and G. Trainin, "Learning vocabulary with apps: from theory to practice," Nebraska Educ. A Student-Led J., vol. 29, pp. 49-69, 2015.

[4] M. Makoe and T. Shandu, "Developing a mobile app for learning english vocabulary in an open distance learning context," Int. Rev. Res. Open Distance Learn., vol. 19, no. 4, pp. 208-221, 2018.

[5] B.-T. Wang, "Designing mobile apps for english vocabulary learning," Int. J. Inf. Educ. Technol., vol. 7, no. 4, pp. 279-283, 2017.

[6] P. Sweeney and C. Moore, "Mobile apps for learning vocabulary," Int. J. Comput. Lang. Learn. Teach., vol. 2, no. 4, pp. 1-16, 2012.

[7] K. Kumar and Y. Lu, "Cloud computing for mobile users: can offloading computation save energy?," in Computer, vol. 43, no. 4, pp. 51-56, April 2010.

[8] A. A. M. Bastina, N. Rama, "Biometric identification and authentication providence using fingerprint for cloud data access," International Journal of Electrical and Computer Engineering, vol. 7, no. 1, pp. 408-416, 2017.

[9] D. K. Kim, "Development of mobile cloud applications using UML," International Journal of Electrical and Computer Engineering, vol. 8, no. 1, pp. 596-604, 2018.

[10] G, Hayardisi, K, B, Seminar, A, Ramadhan, "Analysing signal strength and connection speed in cloud networks for enterprise business intelligence," TELKOMNIKA (Telecommunication, Computing, Electronics and Control), vol. 16, no. 4, pp. 1779-1784, 2018.

[11] R. A. Hasan, M. A. Mohammed, Z. H. Salih, M. A. Ameedeen, N. Ţăpuş, M. N. Mohammed, "HSO: A hybrid swarm optimization algorithm for re-ducing energy consumption in the cloudlets," TELKOMNIKA (Telecommunication, Computing, Electronics and Control), vol. 16, no. 5, pp. 2144-2154, 2018.

[12] A. Ismail, N. A. Jamaludin, S. Zambri, "A review of energy-aware cloud computing surveys," TELKOMNIKA (Telecommunication, Computing, Electronics and Control), vol. 16, no. 6, pp. 2740-2746, 2018.

[13] N. Hegde, S. S. Manvi, "A novel key management protocol for vehicular cloud security," TELKOMNIKA (Telecommunication, Computing, Electronics and Control), vol. 17, no. 2, pp. 857-865, 2019.

[14] A. Bhawiyuga, D. P. Kartikasari, K. Amron, O. B. Pratama, M. W. Habibi, "Architectural design of IoT-cloud computing integration platform," TELKOMNIKA (Telecommunication, Computing, Electronics and Control), vol. 17, no. 3, pp. 1399-1408, 2019.

[15] T. Francis, "A comparison of cloud execution mechanisms fog, edge, and clone cloud computing," International Journal of Electrical and Computer Engineering, vol. 8, no. 6, pp. 4646-4653, 2018.

[16] R.Wishah, M. Al-Nsour, M. Alharafsheh, S. AlKhalaileh, F. Alshbeekat, "Factors influencing cloud computing in telecommunications companies," Indonesian Journal of Electrical Engineering and Computer Science, vol. 12, no. 3, pp. 1334-1339, 2018.

[17] L. Pallavi, A. Jagan, B. T. Rao, "ERMO2 algorithm: an energy efficient mobility management in mobile cloud computing system for 5G heterogeneous networks," International Journal of Electrical and Computer Engineering, vol. 9, no. 3, pp. 1957-1967, 2019.

[18] R. F. Olanrewaju, T. Islam, O. O. Khalifa, F. E. Fajingbesi, "Data in transit validation for cloud computing using cloud-based algorithm detection of injected objects," Indonesian Journal of Electrical Engineering and Computer Science, vol. 10, no. 1, pp. 348-353, 2018.

[19] D. C. Vinutha, G.T. Raju, "An accurate and efficient scheduler for hadoop mapreduce framework," Indonesian Journal of Electrical Engineering and Computer Science, vol. 12, no. 3, pp. 1132-1142, 2018.

[20] A. Satapathy, J. Livingston L. M, "An intelligent framework prototype for monitoring students in virtual classroom," Indonesian Journal of Electrical Engineering and Computer Science, vol. 12, no. 3, pp. 1151-1158, 2018. 
[21] A. Lingayat, R. R. Badre, A. K. Gupta, "Integration of linux containers in openstack: an introspection," Indonesian Journal of Electrical Engineering and Computer Science, vol. 12, no. 3, pp. 1094-1105, 2018.

[22] R. Mahadevan, N. Anbazhagan, "An efficient framework to improve QoS of CSP using enhanced minimal resource optimization based scheduling algorithm," Indonesian Journal of Electrical Engineering and Computer Science, vol. 12, no. 3, pp. 1179-1186, 2018.

[23] G. Panatula, K. S. Kumar, D. E. Geetha, T. V. S. Kumar, "Performance evaluation of cloud service with hadoop for twitter data," Indonesian Journal of Electrical Engineering and Computer Science, vol. 13, no. 1, pp. 392-404, 2019.

[24] N. N. Kulkarni, S. A. Jain, "Checking integrity of data and recovery in the cloud environment," Indonesian Journal of Electrical Engineering and Computer Science, vol. 13, no. 2, pp. 626-633, 2019.

[25] M. R. Prasad, J. Gyani, and P. R. K. Murti, "Mobile cloud computing: implications and challenges," J. Inf. Eng. Appl., vol. 2, no. 7, pp. 7-16, 2012.

[26] P. Pocatilu, F. Alecu, and M. Vetrici, "Using cloud computing for E-learning systems," Recent Adv. Data Networks, Commun. Comput., pp. 54-59, 2009.

[27] Y. Tang, "TF.Learn: TensorFlow's high-level module for distributed machine learning," arXiv preprint arXiv: 1612.04251, 2016.

[28] M. Abadi, P. Barham, J. Chen, Z. Chen, A. Davis, J. Dean, M. Devin, et al., "TensorFlow: A system for large-scale machine learning," in Proceedings of the 12th USENIX Symposium on Operating Systems Design and Implementation (OSDI'16), pp. 265-283, 2016. 